\title{
Febrile Neutropenia in Children with Cancer
}

\author{
Stéphane Paulus and Simon Dobson
}

\section{Introduction}

Febrile neutropenia $(\mathrm{FN})$ is a common complication in children treated for malignancies. In this review, we describe recent advances in several aspects of this topic at the crossroads between oncology and infectious diseases. Although a large amount of information on the subject comes from the adult literature, we have tried to integrate information from paediatric series when this data were available.

\section{Definitions}

A recent article has highlighted the lack of an agreed definition of fever, and the variability amongst units caring for children with cancer (Phillips B., et al., 2007). The Infectious Diseases Society of America 2002 guidelines for the use of antimicrobial agents in neutropenic patients with cancer defines fever as a single oral temperature of $38.3^{\circ} \mathrm{C}$, or $38.0^{\circ} \mathrm{C}$ twice at least $1 \mathrm{~h}$ apart (Hughes et al., 2002). Neutropenia is defined as an absolute neutrophil count (ANC) of less than 500 cells $/ \mu \mathrm{L}$, or less than 1000 cells $/ \mu \mathrm{L}$ in a patient anticipated to rapidly become severely neutropenic after a course of chemotherapy.

\section{Microbiology and Antimicrobials in Febrile Neutropenia}

Febrile neutropenic patients are at high risk of bacterial sepsis. Prompt initiation of empiric antimicrobial therapy has been critical in reducing mortality and morbidity in those patients (Viscoli, 2005). A variety of empiric antibiotic regimens can be used for FN. While published guidelines are useful, they cannot

\footnotetext{
S. Paulus $(\bowtie)$

British Columbia Children's Hospital, 4480 Oak Street, Ambulatory Care Building - Room K4-218, Vancouver, British Columbia, Canada

e-mail: scpaullus@gmail.com
} 
replace a good knowledge of local resistance patterns. Regular surveillance of the local microbiological data is necessary to inform the best choice of first-line empiric therapy (Ammann et al., 2004).

\subsection{Common Pathogens}

Blood cultures are positive in $20-30 \%$ of paediatric or adult patients with FN (Chamberlain et al., 2005; Hann et al., 1997). This is a conservative estimate in view of the frequently inadequate blood volume drawn for blood cultures in paediatric patients (Connell et al., 2007) and the difficulty in recovering some organisms, which can be fastidious to culture (e.g. yeast). Most patients will be colonized by the infecting organisms, usually after initial admission to the hospital, before invasive disease becomes apparent (Walsh et al., 2005). This is important because knowledge of local bacterial flora on a particular unit can inform empiric antibiotic choices. Organisms will then either invade an injured mucosal barrier secondary to chemotherapy (translocation from the oral mucosa or gut) or access the blood stream via the skin, through central venous catheters or skin breakdown. Gut translocation represents a major route of infection, and some authors have advocated the use of non-absorbable antibiotics in order to eradicate the carriage of potential pathogen in the gut (Paulus et al., 2005). However, there is ongoing debate in the literature about the usefulness of this approach and its impact on the potential emergence of multiresistant organisms.

The microbiology of $\mathrm{FN}$ is a constantly changing picture. In the last decade, the focus has shifted towards an increased prevalence of Gram positive organisms, which now represent about $70 \%$ of blood cultures of patients with FN (Hughes et al., 2002). In order of frequency organisms recovered are: coagulase negative staphylococcus, viridans group streptococcus (VGS), Staphylococcus aureus, and Enterococcus faecalis. Gram negative organisms are responsible for most of the mortality associated with sepsis in oncology patients. Most commonly isolated are Escherichia coli, Klebsiella pneumoniae, and Enterobacter spp. Although usually described as a classical pathogen in the context of FN, Pseudomonas aeruginosa prevalence is highly variable from institution to institution. Anaerobic organisms are more commonly associated with intraabdominal sepsis and typhlitis (inflammation of the caecum seen post chemotherapy). Finally, infections with fungal pathogens, such as Candida spp. and Aspergillus spp., play an important role in high-risk patients (HSCT, AML) with persistent severe FN.

\subsection{Empiric Therapy Choices}

To cover the range of pathogens encountered in the setting of $\mathrm{FN}$, the prompt administration of broad spectrum antibiotic therapy is necessary. The use of 
monotherapy versus combination therapy with an aminoglycoside has been much debated in the literature. A Cochrane Collaboration review, evaluating 46 randomized controlled trials, which included 7642 adult and paediatric patients, showed no significant benefit for combination therapy in terms of survival or treatment failure, while adverse events were more common with combination treatment (Paul et al., 2003). The report recommends the adoption of monotherapy with a broad spectrum beta-lactam as the standard of care for the treatment of FN in adult patients. In another recent review summarizing studies and meta-analyses on the empirical antibiotic therapy in high risk patients, different authors draw a similar conclusion regarding the preference for monotherapy (Glasmacher et al., 2005). The paediatric data is more limited at this time, but the gathering evidence seems to show similar efficacy using monotherapy as combination with an aminoglycoside (Agaoglu et al., 2001; Ariffin et al., 2006; Duzova et al., 2001; Hung et al., 2003).

Regimens using a cephalosporin, usually ceftazidime with or without an aminoglycoside, has shown efficacy in the past (Granowetter et al., 1988). Ceftazidime has good activity against $P$. aeruginosa and most Gram negative bacilli. However, its lack of reliable activity for Gram positive organisms such as Streptococcus spp. and S. aureus has been a growing concern in an era of increasing infections with those organisms (Hughes et al., 2002). Ceftriaxone has also been used for empiric cover in FN with success (Ariffin et al., 2001; Charnas et al., 1997). It is to be used with caution because, although it has better coverage of Streptococcus spp., ceftriaxone lacks any activity against $P$. aeruginosa. Additionally, cephalosporins do not have any activity against Enterococcus spp.

Cefipime, a fourth-generation cephalosporin has an expanded spectrum of activity for Gram positive pathogens, with reliable activity for $S$. aureus and VGS while displaying enhanced Gram negative cover, being resistant to Amp-C type $1 \beta$-lactamases produced by an increasing number of Enterobacteriaceae. It lacks activity against Enterococcus faecalis. Cefepime monotherapy has been reported as a feasible option for treatment of childhood cancer patients with FN (Ariffin et al., 2006; Chastagner et al., 2000). In a clinical trial comparing cefipime with ceftazidime monotherapy, it was shown that the addition of vancomycin was required less frequently with cefipime (Owens et al., 2000). Cefipime monotherapy also showed a quicker defervescence, shorter hospitalization, and lower therapy cost when compared with combination of ceftazidime and amikacin (Corapcioglu and Sarper, 2005). However, the Food and Drug Administration (FDA) issued a caution in late 2007 on the use of cefipime in this setting as possibly having a higher all cause mortality than other betalactam antibiotics.

The carbapenems imipenem and meropenem have excellent in vitro activity for Gram positive organisms, Gram negative organisms, and anaerobes. They are active agents against $P$. aeruginos $a$ and are resistant to $\beta$-lactamase-producing organisms. However, there are now increasing concerns regarding the emergence of new carbapenemases in some Gram negative bacilli. There is little 
difference between meropenem and imipenem. Meropenem has better in vitro activity against Gram negatives with minimal inhibitory concentrations (MIC) tenfold lower for most Gram negative organisms compared with imipenem (Chambers, 2005). Conversely, imipenem has better in vitro activity for Gram positive organisms. Meropenem has potential advantages over imipenem with regard to gastrointestinal toxicities and reported lower threshold for the onset of seizure in seriously ill patients with imipenem use (Walsh et al., 2005). Meropenem has been well studied as an agent for children with FN (Cometta et al., 1996; Duzova et al., 2001; Fleischhack et al., 2001) and has demonstrated clinical superiority to ceftazidime and amikacin in a randomized clinical trial (Hung et al., 2003).

The combination of a $\beta$-lactam antibiotic with a $\beta$-lactamase inhibitor has been increasingly popular for use in children. Examples are piperacillin/ tazobactam and ticarcillin/clavulanate. These antibiotics are well suited in the context of FN as they possess a wide spectrum of activity on most Gram positive, negative, and anaerobic organisms. It is important to note that ticarcillin/clavulanate is less active in vitro than piperacillin/tazobactam against Streptococcus spp., E. faecalis, Klebsiella spp., and Pseudomonas spp. (Blondell-Hill, 2006). Piperacillin/tazobactam display a good safety profile and has been shown to be an effective agent in children with FN (Corapcioglu et al., 2006; Fouyssac et al., 2005; Le Guyader et al., 2004).

\section{Practice Surveys}

There are a great variety of approaches when it comes to dealing with febrile neutropenic children not only around the globe, but also from institution to institution within a single country. Definition of fever, empiric regimen of choice, and risk stratification may vary from centre to centre. The lack of unifying approach is of concern and there have been calls for more standardization in the way in which the diagnosis and treatment of children with $\mathrm{FN}$ is approached (Phillips R. et al., 2007). Three practice surveys from the UK, Australasia, and Canada have recently been published and illustrate this issue. On the other hand, this variability of practice should allow for comparisons to be made in future collaborative studies.

A UK survey published in 2007 (Phillips B. et al., 2007) reports on the heterogeneity of the approach for the management of FN. A questionnaire was sent to all of the 21 United Kingdom Children's Cancer Study Group (UKCCSG) assessing local policies and protocols for the management of FN. The definition of fever used in these centres ranged from a persistent temperature of $>37.5^{\circ} \mathrm{C}$ to a single reading of $>39.0^{\circ} \mathrm{C}$. Neutropenia was defined as either an $\mathrm{ANC}<1,<0.75$, or $<0.5$ cells $/ \mu \mathrm{L}$ depending on the unit. A variety of antibiotic combinations were used, the most common consisting of a piperacillin containing antibiotic together with an aminoglycoside compound. Indications for modification of the empiric regimen varied greatly and few centres had a defined endpoint for treatment with an antifungal. Finally, risk stratification 
was undertaken in 11 centres, with six using a policy of reduced intensity therapy in 'low-risk' patient. The UKCCSG is currently working towards the development of a common strategy in the approach of FN patients in the UK.

In a prospective audit undertaken in Australia and New Zealand (Chamberlain et al., 2005), authors reported on the variability in treatment approaches to children with FN. They looked at the management of all cases of FN in nine centres for a period of 2 months. There are no published guidelines for the management of FN in either country. They report 127 episodes of $\mathrm{FN}$, of which a positive blood culture was documented in $30 \%$. There were 18 different first-line antibiotic combinations used, the most popular being a combination of ticarcillin/clavulanic acid and gentamicin. Vancomycin was the most common addition to the empiric regimen. The median length of stay in the hospital was 6 days. Six out of nine centres had a protocol for early discharge in low-risk patients, most commonly on daily ceftriaxone and tobramycin intravenously. Two deaths were recorded in that study period, neither linked to an infectious aetiology.

Reporting on the Canadian experience (Boragina et al., 2007), investigators have focused on the different approach in centres regarding risk stratification and the possible management of patients in an outpatient setting. The survey included 17 centres, 14 of which did offer modified treatment for children considered low risk. The most common antibiotic regimens were a two-drug combination with an aminoglycoside and either an antipseudomonal penicillin or ceftazidime. Four centres had protocols for entirely outpatient management of patients meeting the local criteria of low risk. They used ceftriaxone \pm tobramycin every $24 \mathrm{~h}$. These centres report a high success rate with this approach with more than $80 \%$ of patients being successfully treated as outpatients. However, the lack of agreed consensus on the definition of low risk at presentation leads to most Canadian centres being still reluctant to use strict outpatient management. This chapter underlines the necessity to have large prospective observational studies to derive and validate low-risk criteria, followed by a multicentred clinical trial assessing alternative and traditional treatment in children.

In the following section we will review criteria that have been developed by authors in the adult and paediatric literature to try and establish a risk stratification of FN patients.

\section{Defining Low-Risk and High-Risk Patients}

A focus of recent attention has been the possibility of distinguishing between children at high risk of developing bacterial sepsis, where an aggressive approach is required, and those at low risk, who could be managed at home. In adults, there exists an accepted scoring system for risk prediction, and a number of studies have helped define a low-risk approach to the management of FN using the MASCC (Multinational Association for Supportive Care in Cancer) risk index 
(Innes and Marshall, 2007; Klastersky et al., 2000). This validated index is based on seven independent risk factors present at the onset of FN. These include age, clinical symptoms and severity (hypotension), type of cancer, previous fungal infection, and chronic pulmonary obstructive disease. No such consensus on risk prediction has been reached in children. However, several studies have attempted to develop a risk stratification based on history, physical findings, and laboratory values. Table 1 summarizes recent studies which have looked at risk factors for serious bacterial infection in children with FN.

Other authors have tried to identify particular laboratory markers which would bring high sensitivity and specificity to the question of predicting serious bacterial infection in FN. C-reactive protein (CRP), interleukins (IL-6, IL-8), and procalcitonin (PCT) have all been used either alone or in combination to try to predict the presence or absence of sepsis. In a study of 56 children with a known malignancy who presented with fever and neutropenia, Stryjewski et al. reported that combined CTpr (PCT precursor) $>500 \mathrm{pg} / \mathrm{ml}$ at $24 \mathrm{~h}$ combined with IL-8 $>20 \mathrm{pg} / \mathrm{ml}$ at $48 \mathrm{~h}$ after admission predicted sepsis with $94 \%$ sensitivity and 90\% specificity (Stryjewski et al., 2005). In a study involving 68 episodes of FN, investigators have reported on the superiority of IL-6 and PCT over CRP (Kitanovski et al., 2006). PCT and IL-6 had both an excellent negative predicting value of $97.3 \%$ and $95.6 \%$, respectively, on the day after presentation with FN. Different authors have used a combination of CRP, IL-8, and monocyte chemotactic protein 1- $\alpha$ (MCP-1- $\alpha$ ) measured within $24 \mathrm{~h}$ of the onset of fever. MCP-1- $\alpha$ had the best specificity (92.3\%) and positive predicative value (95\%) (El-Maghraby et al., 2007). It appears that the combination of two or three markers for sepsis holds some promise to help stratify risk in FN patients. However, prospective randomized studies are necessary. One unanswered question is how sensitive does a combination of tests needs to be if management is to be based on the result? In FN patients the stakes are high and both parents and doctors are likely to be reluctant to rely on laboratory results alone.

\section{Outpatient Management for FN}

Children who present with FN and classified as low risk for complication have been increasingly managed with early discharge, or entirely as outpatients with daily re-evaluation. This treatment philosophy has several potential advantages:

- Convenience for children and their families

- Improved quality of life

- Reduction of the incidence of nosocomial infections

- Reduction in the prolonged use of potent wide-spectrum antibiotics

- Reduction in antibiotic-related toxicity

- Reduction of the economic impact of admission to the hospital 


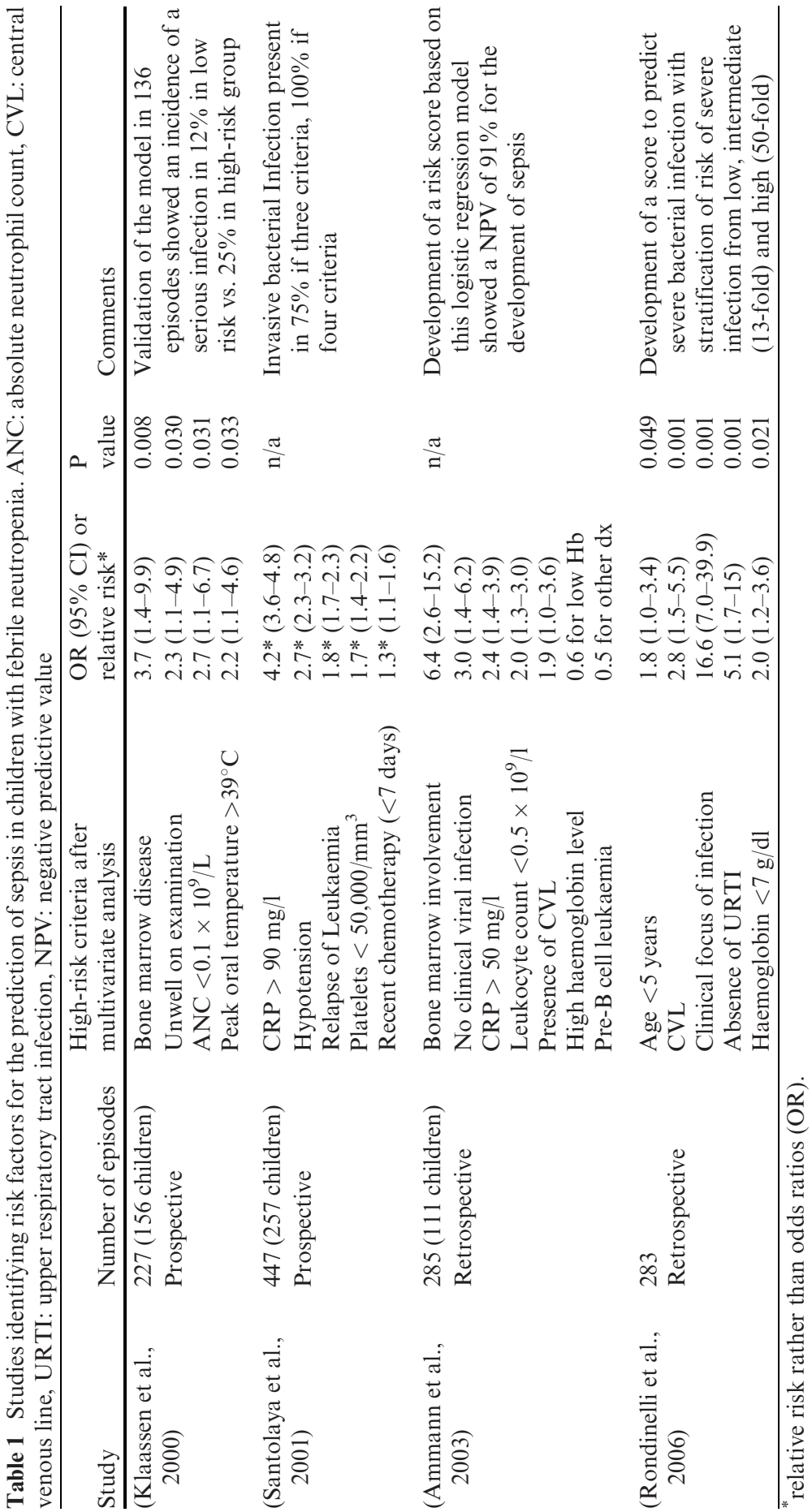


In a report that describes the acceptance of outpatient therapy by doctors and families, Quezada et al. find that there are multiple barriers to the implementation of such protocols. They point out that the medical-exclusion criteria usually adopted for such treatment are stringent resulting in only between onequarter to a third of patients being eligible for outpatient management. Social barriers such as communication issues (language), distance from the hospital, or reluctance from parents or physicians to pursue the strategy can also prevent outpatient management. Nevertheless, this practice has been increasingly popular in the last decade and is the subject of several publications, which are detailed in Table 2. Typical practice consists of a short course of observation (1-24 h) or hospitalization followed by outpatient management with either an intravenous agent such as ceftriaxone or oral ciprofloxacin, along with daily re-evaluation. Although a multicentre trial on risk stratification is necessary to further assess the safety and efficacy of outpatient management in children, it seems to be a reasonable approach at this time in a defined subset of patients at low risk for bacterial sepsis.

\section{Imaging}

The yield of routine chest X-rays is low in asymptomatic neutropenic patients, but an initial X-ray at presentation with FN provides a baseline to further examination (Walsh et al., 2005). It also might reveal some subtle indication of an infectious pneumonic process, which could lead to further imaging using high-resolution CT (HRCT) and possible indication for a broncho-alveolar lavage (BAL).

Patients with persistent FN are at increased risk for invasive fungal disease (IFI) and are usually started on antifungal therapy at 5-7 days of fever. In adult practice, the standard of care is now to perform a CT of the chest $(+/-$ sinuses) at the time of starting antifungal therapy. This practice leads to an earlier diagnosis of IFI, in particular with moulds, such as invasive pulmonary aspergillosis (IPA). In IPA, patients characteristically develop a 'halo-sign' (Fig. 1) on CT early in the first week of the disease (Caillot et al., 1997). In a study of patients with IPA, $95 \%$ of subjects had characteristic halo-sign lesions on HRCT when chest X-ray showed either normal (29\%) or non-specific findings (71\%) (Hauggaard et al., 2002). It is important to stress that the halo-sign is only present in the first week in IPA, and then progresses to be a non-specific infiltrate if the CT is performed at a later stage. Caillot and colleagues have also demonstrated the benefit of using early CT, along with early surgery and antifungals in IPA. Using this approach, they report a cure rate of about $84 \%$, compared with a success rate of $40-50 \%$ usually reported in the literature (Caillot et al., 1997, 2001).

By contrast, the paediatric literature has fewer reports on the use of early CT in FN. In a retrospective review of CT in 109 episodes of prolonged FN in 


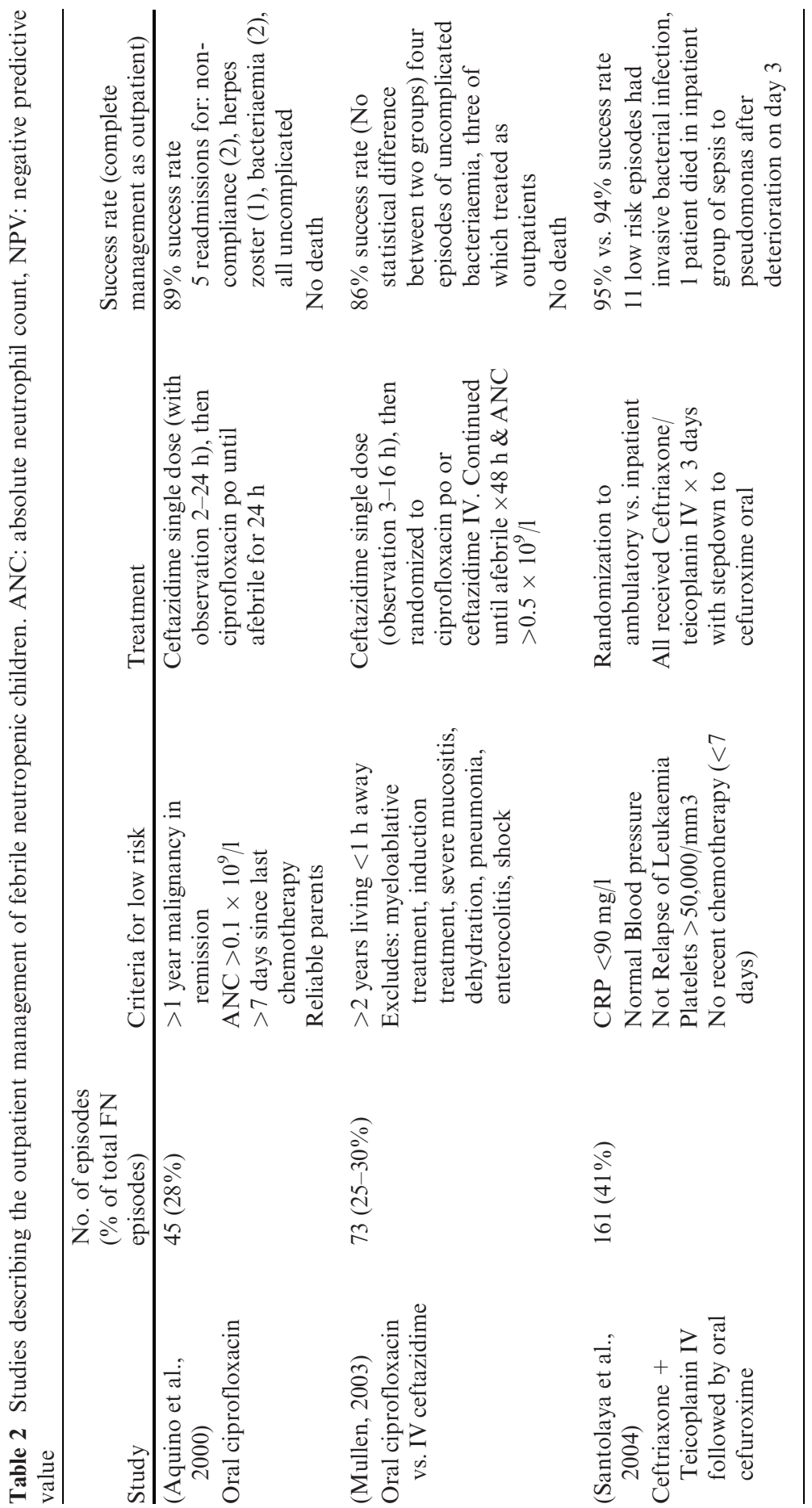




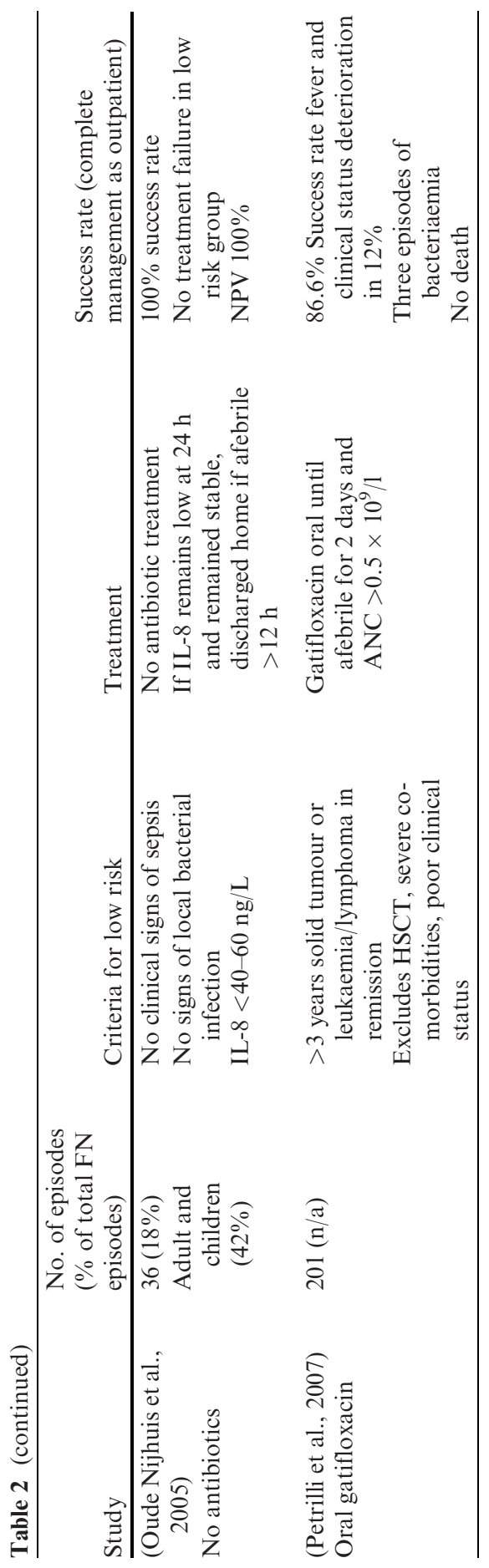


Fig. 1 CT scan of the chest in a 12-year-old patient with AML and a history of long-standing neutropenia with 5 days of fever. The lesion in the right lung displays the characteristic 'halo-sign' feature of a macronodule surrounded by an area with a ground glass appearance. The patient was treated with intravenous followed by oral voriconazole with good clinical response

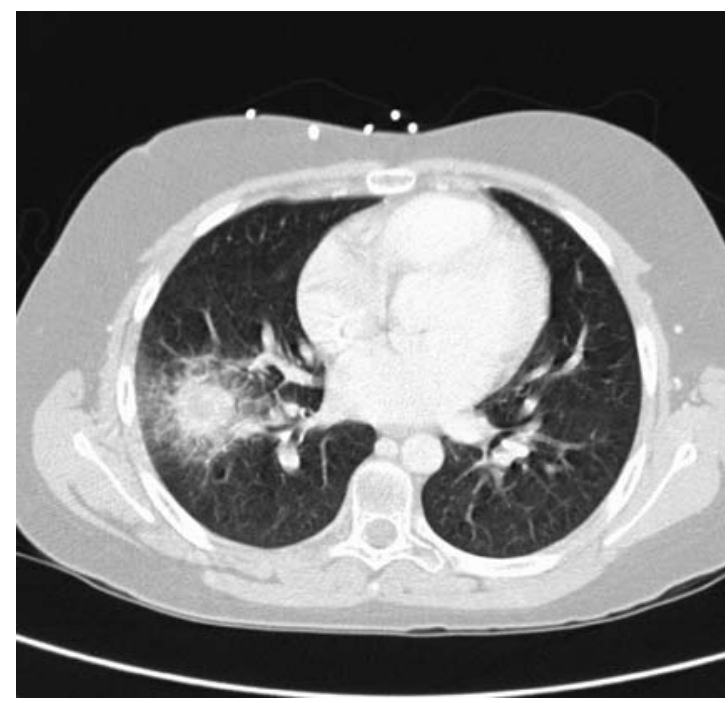

paediatric patients, investigators from the University of Washington, Seattle, emphasize the diagnostic utility of performing chest CT (Archibald et al., 2001). In this review, $49 \%$ of children had CT abnormalities, which led to the modification of therapy in a third of patients. In a single institution review of 10 years of invasive aspergillosis in children, investigators from the UK document the finding on imaging in 27 patients with documented Aspergillus infection (Thomas et al., 2003). The X-ray finding had great variability including segmental and multilobar consolidations, perihilar infiltrates, and nodular masses. CT examinations were available in eight cases, and had been performed late in the disease. None had halo-signs and two children had presence of cavitating nodules. The authors acknowledge that this is a reflection of the late stage of the disease in which the patients were scanned in an era where CT was not so readily available. At the conclusion of their article, they recommend performing CT early (at 5-7 days of fever) in order to find the characteristic halo-sign.

We believe that the reluctance to CT high risk patients for IPA because of exposure to radiation must be balanced with the improved success rates in diagnosing and treating this illness in the early stages. Confirmation of the diagnosis by percutaneous biopsy has shown great specificity and susceptibility in paediatric patients (Hoffer et al., 2001).

\section{The Role of Respiratory Viruses}

Respiratory infections due to viruses are ubiquitous in children. The role played by viral infections in the context of FN is not well established. Direct immunofluorescence techniques (DFA) performed on naso-pharyngeal washings 
(NPW) can identify most common respiratory viruses. This allows for a rapid and reliable diagnosis of viral URTI and has been a useful tool for paediatricians looking after immunocompetent children. Using this technique, reports have described that between $25 \%$ and $37 \%$ of patients with $\mathrm{FN}$ have viral respiratory infections (Arola et al., 1995; Tager et al., 2006). However, studies looking at the role played by viruses in FN are limited and no large trial has been published to date. In addition, little is known about the interaction of viral pathogens with colonizing bacteria in the respiratory tract of FN patients. New molecular diagnostic techniques now available for testing of BAL or NPW might lead to further information on the prevalence and role of viruses in FN. The usual technique to collect NPW involves flushing a small volume of saline into a nostril with the head tipped back followed by the insertion of a soft narrow bore catheter into the nasopharynx and applying suction. There is reluctance to do this in oncology patients, not least because of the fear of inducing bleeding in thrombocytopenic children. Alternative techniques of nasal swabbing or of tipping nasally inserted saline back into a paper cup have been used in patients with some degree of success (Heikkinen et al., 2002).

\section{Emerging Pathogens}

\subsection{Viridans Group Streptococcus}

VGS are alpha-haemolytic Gram positive cocci belonging that are part of the normal flora of the oral cavity, upper respiratory tract, and gastrointestinal system. In the last 15 years, VGS has become a leading cause of bacteriaemia and sepsis in the immunocompromised host, particularly in children undergoing chemotherapy for AML and post haematopoietic stem cell transplantation (HSCT). In two large multi-institutional studies of children with AML, VGS represented 22-25\% of all bacteriaemic events (Gamis et al., 2000; Lehrnbecher et al., 2004). In a review of 36 cases of VGS bacteriaemia from 1991 to 2000 at St. Jude Hospital, Memphis, TN, a recrudescence of cases linked to a change of protocol using increased doses of cytosine arabinoside was noted (Okamoto et al., 2003). High-dose cytosine arabinoside has since been linked to the development of VGS sepsis by other authors (Lehrnbecher et al., 2004; Paganini et al., 2003). It is unclear if this is secondary to a direct effect of this chemotherapeutic agent or is the indirect result of the development of mucositis and the prolonged period of neutropenia induced by this agent. Patients with VGS bacteriaemia often present with septic shock and acute respiratory distress syndrome (ARDS). In the report by Okamoto et al., patients were febrile for a median of 15 days; $64 \%$ of patients were admitted to ICU, 33\% experienced hypotension, and $28 \%$ had ARDS. A higher-thanexpected proportion of these patients $(18 \%)$ subsequently developed an IFI (one of whom died of IPA) during the same febrile episode. No patients died 
primarily of VGS-associated septic shock. The prolonged duration of the fever and the associated symptoms are not clearly explained, as most of the blood cultures become rapidly negative. Some authors have suggested a role for an inflammatory response triggered by the organism leading to a degree of cytokine release dysregulation (Ihendyane et al., 2004). However, no causative toxin has been demonstrated to be present in VSSS.

The increase in VGS septic episodes has led to an increase in the use of vancomycin as part of the empiric antibiotic regimen in FN. VGS resistance to penicillin is variable reported from 4 to $14 \%$, with intermediate susceptibility reported between 14 and 64\% (Bruckner and Gigliotti, 2002). Amongst $\beta$-lactam antibiotics, ceftazidime has been reported as having the least activity in vitro, with mean MIC reported 15 -folds higher than penicillin (Kennedy et al., 2001). It is therefore a reasonable proposition to start vancomycin empirically in AML patients who present with sepsis, before identification and susceptibilities are fully known.

\section{$9.2 \beta$-Lactamase-Producing Organisms}

$\beta$-Lactamase-producing organisms are an increasing concern in oncologyrelated infections. This occurs either through plasmid transfer, such as in extended spectrum $\beta$-lactamase-producing organisms (ESBL) or though induction of a chromosomally encoded $\beta$-lactamase such as in class $C$ cephalosporinase (AmpC). These two mechanisms constitute the most clinically relevant mechanisms of resistance in Gram negative bacilli. ESBL are mostly found in $E$. coli and $K$. pneumoniae. In a study looking at the prevalence of ESBL in $K$. pneumoniae bloodstream infection in a children's oncology unit in Malaysia, authors describe a prevalence of ESBL-producing bacteria in up to $50 \%$ of clinical isolates (Ariffin et al., 2000). Predisposing factors were recent exposure to a third-generation cephalosporin-containing regimen and a hospital stay of 2 weeks or more. AmpC-producing Enterobacteriaciae, such as Serretia spp. or Acinetobacter spp., are inducible and can even develop resistance while the patient is on large-spectrum antibiotics. There is variability between antibiotics regarding their potential to induce this AmpC enzyme, as well as their lability to its production. First-generation cephalosporin ceftazidime and carbapenems are good inducers, but only carbapenems are not labile to the enzyme produced. The incidence of these various resistant organisms is variable from institution to institution and knowledge of local microbiological data is key in making decisions about appropriate empiric antimicrobial therapy protocols.

\subsection{Vancomycin-Resistant Enterococcus}

Vancomycin-resistant enterococci (VRE) have been a concern in an era where some institutions have been using vancomycin or teicoplanin as part of an 
empiric regimen protocol. Other risk factors include duration of neutropenia and antibiotic therapy, with ceftazidime or amikacin in particular (Nourse et al., 1998). Outbreaks with VRE have been described in paediatric oncology units with associated deaths (Gray and George, 2000). Patient-to-patient transmission on an oncology unit is an important recognized factor in the development of outbreaks. Therefore, barrier isolation associated with a restricted used of glycopeptides have been key in decreasing colonization of patients at risk (Nourse et al., 1998; Schuster et al., 1998). The emergence of VRE also illustrates the difficulty of trading off the risk to the individual patient and society (or at least the oncology unit). A balance is required to give maximal benefit of broad-spectrum coverage to the individual and the wider benefit to the unit as a whole by limiting the use of unnecessary agents and therefore avoid the spread of organisms such as VRE.

\section{Best Practice}

In this final section of the chapter we will try to offer a practical way to approach patients with FN (Fig. 2). A standardized approach starts with a clear and accepted definition of fever and neutropenia. We define fever as an oral temperature of $38.3^{\circ} \mathrm{C}$, or $38.0^{\circ} \mathrm{C}$ twice at least $1 \mathrm{~h}$ apart. Neutropenia is defined as an absolute neutrophil count (ANC) of less than 1000 cells $/ \mu \mathrm{L}$. Many patients are 'high risk' for the development of sepsis and invasive bacterial disease and are not eligible for outpatient therapy. Those are usually patients undergoing intense chemotherapy such as with AML on relapse protocols or post-HSCT. A recent episode of proven bacterial sepsis, expected neutropenia for more than 1 week, severe mucositis or the clinical suspicion of typhlitis also exclude patients for management as outpatients. Inclusion criteria for 'low risk' management usually include a clinically well child with viral symptoms, a CRP $<10 \mathrm{mg} / \mathrm{L}$ (other markers such as IL-8 or PCT can be used) and most importantly reliable parents with easy access to the hospital. The use of DFA on NPW samples can also help the clinician to determine if the episode has a viral aetiology. It is possible to manage low-risk patients with daily re-evaluation and intravenous ceftriaxone every $24 \mathrm{~h}$ (or oral ciprofloxacin) until blood cultures are negative at $48-72 \mathrm{~h}$.

In moderate and high-risk patients, one approach is to admit to the hospital for therapy with a broad-spectrum agent such as piperacillin-tazobactam, cefipime, or meropenem. The addition of an aminoglycoside to the empiric regimen is still commonplace in paediatric practice, but as discussed earlier, there is now little evidence to support its use. If an aminoglycoside is used, the choice of agent depends on the local microbiological data. In institutions where Enterococcus spp. is a predominant pathogen, gentamicin is considered a better agent owing to its synergistic properties when given along with $\beta$-lactams. If $P$. aeruginosa infections are predominant, the use of tobramycin may be a better 


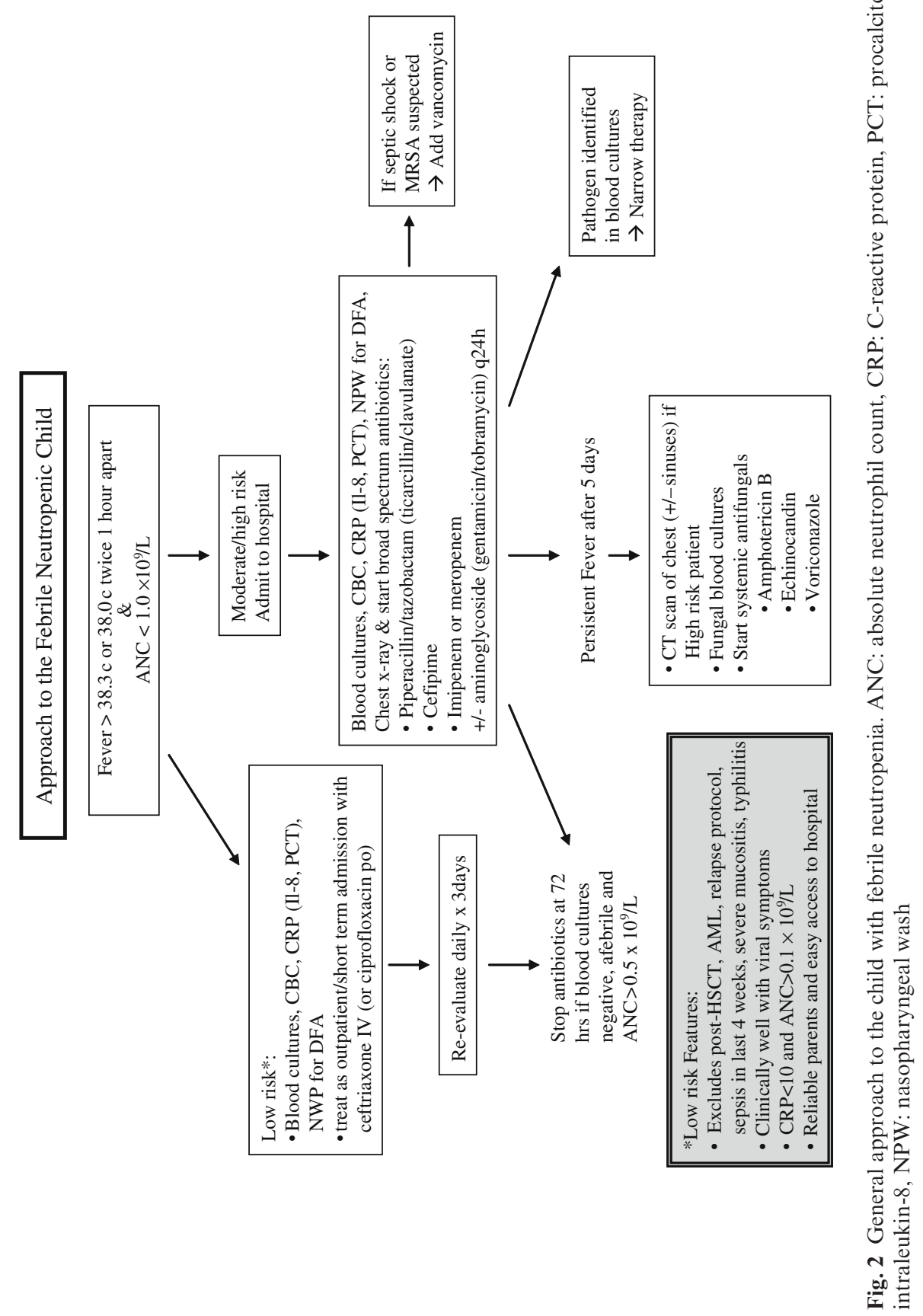


choice. In patients presenting with septic shock, especially in patients with AML (at risk for VGS sepsis), or in patients with suspected MRSA sepsis (e.g. previously colonized or with tunnel infection), the empiric addition of vancomycin is recommended.

The approach to management of suspected IFI is beyond the scope of this review. However, it is important to mention that patients with $\mathrm{FN}$ for 5 days or more should be assessed for the possibility of invasive fungal disease. Fungal blood culture should be drawn and a HRCT of the chest ( $+/-$ sinuses) should be performed in high risk patients (AML, ALL relapse, Post HSCT) prior to the start of antifungal therapy with either amphotericin B, a widespectrum triazole, or an echinocandin. Finally, it is important to remind the clinician of the necessity, upon identification of an aetiological pathogen, to review, and, if possible, narrow the spectrum of antibiotic therapy.

\section{Summary}

- There is a need for increased consensus in the definition of fever and neutropenia, the approach to risk stratification (including outpatient therapy and early discharge) and choices of empiric antimicrobial therapy in children.

- There has been an increased incidence of Gram positive infection in FN patients, in particular with VGS in patient with AML. However, Gram negative bacteria are still responsible for most of the mortality associated with FN.

- Piperacillin/tazobactam, cefipime, or meropenem are all effective first-choice antimicrobial monotherapy in FN. There is no good evidence for adding an aminoglycoside compound to the initial empiric therapy regimen.

- Following local microbiological data is of utmost importance in choosing the right empiric antimicrobial regimen for a particular institution.

- Outpatient management of a well-defined subset of low-risk patient for bacterial invasive infection with intravenous ceftriaxone or oral ciprofloxacin and daily re-evaluation is possible.

- Early CT of the chest (after 5-7 days of FN) in high-risk patients is essential to make a prompt diagnosis of pulmonary aspergillosis and improve outcome.

\section{References}

Agaoglu L., Devecioglu O., Anak S., Karakas Z., Yalman N., Biner B., Eryilmaz E., Goksan B., Unuvar A., Agirbasli H., Can M., Bilgen H.,Gedikoglu G. (2001) Cost-effectiveness of cefepime plus netilmicin or ceftazidime plus amikacin or meropenem monotherapy in febrile neutropenic children with malignancy in Turkey. J Chemother 13: 281-287 
Ammann R. A., Aebi C., Hirt A., Luthy A. R. (2004) Fever in neutropenia in children and adolescents: evolution over time of main characteristics in a single center, 1993-2001. Support Care Cancer 12: 826-832

Ammann R. A., Hirt A., Luthy A. R., Aebi C. (2003) Identification of children presenting with fever in chemotherapy-induced neutropenia at low risk for severe bacterial infection. Med Pediatr Oncol 41: 436-443

Aquino V. M., Herrera L., Sandler E. S., Buchanan G. R. (2000) Feasibility of oral ciprofloxacin for the outpatient management of febrile neutropenia in selected children with cancer. Cancer 88: 1710-1714

Archibald S., Park J., Geyer J. R., Hawkins D. S. (2001) Computed tomography in the evaluation of febrile neutropenic pediatric oncology patients. Pediatr Infect Dis $J$ 20: $5-10$

Ariffin H., Ai C. L., Lee C. L., Abdullah W. A. (2006) Cefepime monotherapy for treatment of febrile neutropenia in children. $J$ Paediatr Child Health 42: 781-784

Ariffin H., Arasu A., Mahfuzah M., Ariffin W. A., Chan L. L., Lin H. P. (2001) Single-daily ceftriaxone plus amikacin versus thrice-daily ceftazidime plus amikacin as empirical treatment of febrile neutropenia in children with cancer. J Paediatr Child Health 37: 38-43

Ariffin H., Navaratnam P., Mohamed M., Arasu A., Abdullah W. A., Lee C. L., Peng L. H. (2000) Ceftazidime-resistant Klebsiella pneumoniae bloodstream infection in children with febrile neutropenia. Int J Infect Dis 4: 21-25

Arola M., Ruuskanen O., Ziegler T., Salmi T. T. (1995) Respiratory virus infections during anticancer treatment in children. Pediatr Infect Dis J 14: 690-694

Blondell-Hill E., Bug and Drugs (4th Ed.), p. 37, Capital Health, Edmonton, AB (2006)

Boragina M., Patel H., Reiter S., Dougherty G. (2007) Management of febrile neutropenia in pediatric oncology patients: A Canadian survey. Pediatr Blood Cancer 48: 521-526

Bruckner L., Gigliotti F. (2002) Alpha-hemolytic streptococcal infections among immunocompromised hosts: Increasing incidence, severity and antibiotic resistance. Pediatr Infect Dis J 21: 343-345

Caillot D., Casasnovas O., Bernard A., Couaillier J. F., Durand C., Cuisenier B., Solary E., Piard F., Petrella T., Bonnin A., Couillault G., Dumas M., Guy H. (1997) Improved management of invasive pulmonary aspergillosis in neutropenic patients using early thoracic computed tomographic scan and surgery. J Clin Oncol 15: 139-147

Caillot D., Couaillier J. F., Bernard A., Casasnovas O., Denning D. W., Mannone L., Lopez J., Couillault G., Piard F., Vagner O., Guy H. (2001) Increasing volume and changing characteristics of invasive pulmonary aspergillosis on sequential thoracic computed tomography scans in patients with neutropenia. J Clin Oncol 19: 253-259

Chamberlain J. D., Smibert E., Skeen J., Alvaro F. (2005) Prospective audit of treatment of paediatric febrile neutropenia in Australasia. J Paediatr Child Health 41: 598-603

Chambers H.; "Other B-Lactams Antibiotics" in Principles and Practice of Infectious Diseases p. 313, Elsevier Churchill Livingstone (2005)

Charnas R., Luthi A. R., Ruch W. (1997) Once daily ceftriaxone plus amikacin vs. three times daily ceftazidime plus amikacin for treatment of febrile neutropenic children with cancer. Writing Committee for the International Collaboration on Antimicrobial Treatment of Febrile Neutropenia in Children. Pediatr Infect Dis $J$ 16: 346-353

Chastagner P., Plouvier E., Eyer D., Plesiat P., Lozniewski A., Sommelet D. (2000) Efficacy of cefepime and amikacin in the empiric treatment of febrile neutropenic children with cancer. Med Pediatr Oncol 34: 306-308

Cometta A., Calandra T., Gaya H., Zinner S. H., de Bock R., Del Favero A., Bucaneve G., Crokaert F., Kern W. V., Klastersky J., Langenaeken I., Micozzi A., Padmos A., Paesmans M., Viscoli C., Glauser M. P. (1996) Monotherapy with meropenem versus combination therapy with ceftazidime plus amikacin as empiric therapy for fever in granulocytopenic patients with cancer. The International Antimicrobial Therapy Cooperative Group of the European Organization for Research and Treatment of Cancer and 
the Gruppo Italiano Malattie Ematologiche Maligne dell'Adulto Infection Program. Antimicrob Agents Chemother 40: 1108-1115

Connell T. G., Rele M., Cowley D., Buttery J. P., Curtis N. (2007) How reliable is a negative blood culture result? Volume of blood submitted for culture in routine practice in a children's hospital. Pediatrics 119: 891-896

Corapcioglu F., Sarper N. (2005) Cefepime versus ceftazidime plus amikacin as empirical therapy for febrile neutropenia in children with cancer: A prospective randomized trial of the treatment efficacy and cost. Pediatr Hematol Oncol 22: 59-70

Corapcioglu F., Sarper N., Zengin E. (2006) Monotherapy with piperacillin/tazobactam versus cefepime as empirical therapy for febrile neutropenia in pediatric cancer patients: A randomized comparison. Pediatr Hematol Oncol 23: 177-186

Duzova A., Kutluk T., Kanra G., Buyukpamukcu M., Akyuz C., Secmeer G., Ceyhan M. (2001) Monotherapy with meropenem versus combination therapy with piperacillin plus amikacin as empiric therapy for neutropenic fever in children with lymphoma and solid tumors. Turk J Pediatr 43: 105-109

El-Maghraby S. M., Moneer M. M., Ismail M. M., Shalaby L. M., El-Mahallawy H. A. (2007) The diagnostic value of c-reactive protein, interleukin-8. and monocyte chemotactic protein in risk stratification of febrile neutropenic children with Hematologic malignancies. J. Pediatr Hematol Oncol 29: 131-136

Fleischhack G., Hartmann C., Simon A., Wulff B., Havers W., Marklein G., Hasan C., Bode U. (2001) Meropenem versus ceftazidime as empirical monotherapy in febrile neutropenia of paediatric patients with cancer. $J$ Antimicrob Chemother 47: 841-853

Fouyssac F., Salmon A., Mansuy L., Schmitt C., Bordigoni P., Chastagner P. (2005) Treatment of febrile neutropenia episodes in children, with a piperacillin-tazobactam and netilmicin combination. Medecine Et Maladies Infectieuses 35: 357-362

Gamis A. S., Howells W. B., DeSwarte-Wallace J., Feusner J. H., Buckley J. D., Woods W. G. (2000) Alpha hemolytic streptococcal infection during intensive treatment for acute myeloid leukemia: a report from the Children's cancer group study CCG-2891. J Clin Oncol 18: $1845-1855$

Glasmacher A., von Lilienfeld-Toal M., Schulte S., Hahn C., Schmidt-Wolf I. G., Prentice A. (2005) An evidence-based evaluation of important aspects of empirical antibiotic therapy in febrile neutropenic patients. Clin Microbiol Infect 11 Suppl 5: 17-23

Granowetter L., Wells H., Lange B. J. (1988) Ceftazidime with or without vancomycin vs. cephalothin, carbenicillin and gentamicin as the initial therapy of the febrile neutropenic pediatric cancer patient. Pediatr Infect Dis J 7: 165-170

Gray J. W., George R. H. (2000) Experience of vancomycin-resistant enterococci in a children's hospital. $J$ Hosp Infect 45: 11-18

Hann I., Viscoli C., Paesmans M., Gaya H., Glauser M. (1997) A comparison of outcome from febrile neutropenic episodes in children compared with adults: results from four EORTC studies. International Antimicrobial Therapy Cooperative Group (IATCG) of the European Organization for Research and Treatment of Cancer (EORTC). $\mathrm{Br} J$ Haematol 99: 580-588

Hauggaard A., Ellis M., Ekelund L. (2002) Early chest radiography and CT in the diagnosis, management and outcome of invasive pulmonary aspergillosis. Acta Radiol 43: 292-298

Heikkinen T., Marttila J., Salmi A. A., Ruuskanen O. (2002) Nasal swab versus nasopharyngeal aspirate for isolation of respiratory viruses. J Clin Microbiol 40: 4337-4339

Hoffer F. A., Gow K., Flynn P. M., Davidoff A. (2001) Accuracy of percutaneous lung biopsy for invasive pulmonary aspergillosis. Pediatr Radiol 31: 144-152

Hughes W. T., Armstrong D., Bodey G. P., Bow E. J., Brown A. E., Calandra T., Feld R., Pizzo P. A., Rolston K. V. I., Shenep J. L., Young L. S. (2002) 2002 guidelines for the use of antimicrobial agents in neutropenic patients with cancer. Clin. Infect. Dis. 34: 730-751

Hung K. C., Chiu H. H., Tseng Y. C., Wang J. H., Lin H. C., Tsai F. J., Peng C. T. (2003) Monotherapy with meropenem versus combination therapy with ceftazidime plus 
amikacin as empirical therapy for neutropenic fever in children with malignancy. J Microbiol Immunol Infect 36: 254-259

Ihendyane N., Sparrelid E., Wretlind B., Remberger M., Andersson J., Ljungman P., Ringden O., Normark B. H., Allen U., Low D. E., Norrby-Teglund A. (2004) Viridans streptococcal septicaemia in neutropenic patients: role of proinflammatory cytokines. Bone Marrow Transplant 33: 79-85

Innes H., Marshall E. (2007) Outpatient therapy for febrile neutropenia. Curr Opin Oncol 19: 294-298

Kennedy H. F., Gemmell C. G., Bagg J., Gibson B. E., Michie J. R. (2001) Antimicrobial susceptibility of blood culture isolates of viridans streptococci: relationship to a change in empirical antibiotic therapy in febrile neutropenia. J Antimicrob Chemother 47: 693-696

Kitanovski L., Jazbec J., Hojker S., Gubina M., Derganc M. (2006) Diagnostic accuracy of procalcitonin and interleukin-6 values for predicting bacteremia and clinical sepsis in febrile neutropenic children with cancer. Eur J Clin Microbiol Infect Dis 25: 413-415

Klaassen R. J., Goodman T. R., Pham B., Doyle J. J. (2000) "Low-risk" prediction rule for pediatric oncology patients presenting with fever and neutropenia. $J$ Clin Oncol 18: 1012-1019

Klastersky J., Paesmans M., Rubenstein E. B., Boyer M., Elting L., Feld R., Gallagher J., Herrstedt J., Rapoport B., Rolston K., Talcott J. (2000) The Multinational Association for Supportive Care in Cancer risk index: A multinational scoring system for identifying low-risk febrile neutropenic cancer patients. J Clin Oncol 18: 3038-3051

Le Guyader N., Auvrignon A., Vu-Thien H., Portier E., Tabone M. D., Leverger G. (2004) Piperacillin-tazobactam and netilmicin as a safe and efficacious empirical treatment of febrile neutropenic children. Support Care Cancer 12: 720-724

Lehrnbecher T., Varwig D., Kaiser J., Reinhardt D., Klingebiel T., Creutzig U. (2004) Infectious complications in pediatric acute myeloid leukemia: analysis of the prospective multi-institutional clinical trial AML-BFM 93. Leukemia 18: 72-77

Mullen C. A. (2003) Ciprofloxacin in treatment of fever and neutropenia in pediatric cancer patients. Pediatric Infectious Disease Journal 22: 1138-1142

Nourse C., Murphy H., Byrne C., O'Meara A., Breatnach F., Kaufmann M., Clarke A., Butler K. (1998) Control of a nosocomial outbreak of vancomycin resistant Enterococcus faecium in a paediatric oncology unit: risk factors for colonisation. Eur J Pediatr 157: 20-27

Okamoto Y., Ribeiro R. C., Srivastava D. K., Shenep J. L., Pui C. H., Razzouk B. I. (2003) Viridans streptococcal sepsis: clinical features and complications in childhood acute myeloid leukemia. J Pediatr Hematol Oncol 25: 696-703

Oude Nijhuis C., Kamps W. A., Daenen S. M., Gietema J. A., van der Graaf W. T., Groen H. J., Vellenga E., Ten Vergert E. M., Vermeulen K. M., de Vries-Hospers H. G., de Bont E. S. (2005) Feasibility of withholding antibiotics in selected febrile neutropenic cancer patients. $J$ Clin Oncol 23: 7437-7444

Owens R. C., Owens C. A., Holloway W. J. (2000) Reduction in Vancomycin consumption in patients with fever and neutropenia [abstract 458]. Clin. Infect. Dis. 31: 291

Paganini H., Staffolani V., Zubizarreta P., Casimir L., Lopardo H., Luppino V. (2003) Viridans streptococci bacteraemia in children with fever and neutropenia: a case-control study of predisposing factors. Eur J Cancer 39: 1284-1289

Paul M., Soares-Weiser K., Grozinsky S., Leibovici L. (2003) Beta-lactam versus beta-lactamaminoglycoside combination therapy in cancer patients with neutropaenia. Cochrane Database Syst Rev 3: CD003038

Paulus S. C., van Saene H. K. F., Hemsworth S., Hughes J., Ng A., Pizer B. L. (2005) A prospective study of septicaemia on a paediatric oncology unit: A three-year experience at The Royal Liverpool Children's Hospital, Alder Hey, UK. Eur J Cancer 41: 2132-2140

Petrilli A., Altruda Carlesse F., Alberto Pires Pereira C. (2007) Oral gatifloxacin in the outpatient treatment of children with cancer fever and neutropenia. Pediatr Blood Cancer 49: $682-686$ 
Phillips B., Selwood K., Lane S. M., Skinner R., Gibson F., Chisholm J. C. (2007) Variation in policies for the management of febrile neutropenia in United Kingdom Children's Cancer Study Group centres. Arch Dis Child 92: 495-498

Phillips R., Skinner R., Chisholm J. C. (2007) Treating low-risk febrile neutropenia: Jenny's story. Arch Dis Child 92: 7-8

Rondinelli P. I. P., Ribeiro K. D. B., de Camargo B. (2006) A proposed score for predicting severe infection complications in children with chemotherapy-induced febrile neutropenia. $J$ Pediatr Hematol Oncol 28: 665-670

Santolaya M. E., Alvarez A. M., Aviles C. L., Becker A., Cofre J., Cumsille M. A., O'Ryan M. L., Paya E., Salgado C., Silva P., Tordecilla J., Varas M., Villarroel M., Viviani T., Zubieta M. (2004) Early hospital discharge followed by outpatient management versus continued hospitalization of children with cancer, fever, and neutropenia at low risk for invasive bacterial infection. J Clin Oncol 22: 3784-3789

Santolaya M. E., Alvarez A. M., Becker A., Cofre J., Enriquez N., O'Ryan M., Paya E., Pilorget J., Salgado C., Tordecilla J., Varas M., Villarroel M., Viviani T., Zubieta M. (2001) Prospective, multicenter evaluation of risk factors associated with invasive bacterial infection in children with cancer, neutropenia, and fever. J Clin Oncol 19: 3415-3421

Schuster F., Graubner U. B., Schmid I., Weiss M., Belohradsky B. H. (1998) Vancomycinresistant-enterococci - colonization of 24 patients on a pediatric oncology unit. Klin Padiatr 210: 261-263

Stryjewski G. R., Nylen E. S., Bell M. J., Snider R. H., Becker K. L., Wu A., Lawlor C., Dalton H. (2005) Interleukin-6, interleukin-8, and a rapid and sensitive assay for calcitonin precursors for the determination of bacterial sepsis in febrile neutropenic children. Pediatr Crit Care Med 6: 129-135

Tager F. M., Zolezzi R. P., Folatre B. I., Navarrete C. M., Rojas P. J. (2006) Respiratory virus infections in children with acute lymphoblastic leukemia and febrile neutropenia: a prospective study. Rev Chilena Infectol 23: 118-123

Thomas K. E., Owens C. M., Veys P. A., Novelli V., Costoli V. (2003) The radiological spectrum of invasive aspergillosis in children: a 10-year review. Pediatr Radiol 33: 453-460

Viscoli C., Castagnola E. "Prophylaxis and Empirical Therapy for Infection in Cancer Patients" in Principles and Practice of Infectious Diseases (6th Ed), pp. 3442-3462, Elsevier Churchill Livingstone (2005).

Walsh T. J., Groll, A., Gonzalez C., Pizzo, P. "Infectious Complications in Pediatric Cancer Patients" in Principles and Practice of Pediatric Oncology (5th Ed.), pp. 1269-1329, Lippincott Williams \& Wilkins (2005) 